\title{
Negative- $\mu$ regime in the ac magnetic response of superconductor nanoshells
}

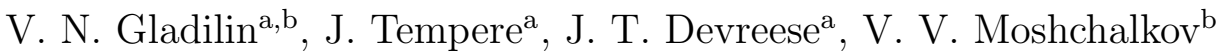 \\ ${ }^{a} T Q C$ - Theory of Quantum and Complex Systems, Universiteit Antwerpen, \\ Universiteitsplein 1, B-2610 Antwerpen, Belgium \\ ${ }^{b}$ INPAC - Institute for Nanoscale Physics and Chemistry, Katholieke Universiteit \\ Leuven, Celestijnenlaan 200D, B-3001 Leuven, Belgium
}

\begin{abstract}
The time-dependent Ginzburg-Landau formalism is applied to analyze the vortex states and vortex dynamics in superconducting spherical nanoshells, subjected to mutually perpendicular strong dc and weak ac magnetic fields. We demonstrate that nonuniformity of the shell thickness can dramatically affect the ac magnetic response of a 3D array of superconducting nanoshells. Remarkably, this response is strongly influenced not only by the relevant geometric and material parameters and the ac-field frequency but also by the magnitude of the applied dc field: by changing this field the real part of the effective ac magnetic permeability can be tuned from positive values significantly larger than one down to negative values.
\end{abstract}

Keywords:

A. Superconductors; A. Nanoshells; D. Vortices; D. Magnetic permeability

\section{Introduction}

Over the last decade, advances in nanotechnology have inspired experimental and theoretical studies of vortex behaviour in superconductors with 
curvilinear surfaces: hollow cylinders and curved stripes [1, 2, 3, 4, 4, 5, 6], ferromagneticsuperconducting coreshell structures[7], superconducting spheres [8, 9, 10, 11] and spherical nanoshells [12, 13, 14, 15], including those with a magnetic dipole inside[16, 17]. Nanoshells are hybrid nanostructures, which typically consist of a dielectric core (usually a silicon oxide nanograin), coated with a thin layer of metal [18, 19]. When the metal in its bulk form is a superconductor, the nanoshell below the critical temperature will also exhibit superconductivity in the thin shell around the insulating core. It has been shown that those nanoshells allow the coexistence of a Meissner state and a vortex state in equilibrium on one and the same superconducting layer [15]. In superconducting spherical nanoshells the surface curvature and the applied homogeneous magnetic field lead to a Magnus-Lorentz force, which pushes the vortices and antivortices[20] towards the opposite poles of the shell. The same effect can be expected in the absence of any external magnetic field for spherical superconductor-ferromagnet nanoshells with uniformly magnetized core. This can be considered as an effective pinning of vortices and antivortices at the poles, which strongly affects both the equilibrium distributions of vortices and their dynamics [12, 13, 14, 15].

In this Communication the vortex dynamics in superconducting nanoshells is investigated for the case when in addition to a (strong) dc magnetic field, which provides vortex pinning, also a weak ac magnetic field is present. In particular, we analyze the ac magnetic response of nanoshells with nonuniform thickness of the superconducting shell. We show that the effective ac magnetic permeability of nanoshells, arranged in a 3D array, is very sensitive to the applied dc magnetic field. For one and the same array of nanoshells, 
the real part of the ac magnetic permeability at a given frequency can be tuned from relatively large ("superparamagnetic") positive values at relatively high dc fields to negative values at lower dc fields.

\section{Model}

A sketch of a spherical nanoshell with thickness $d$ and radius $R$ is shown in Fig. 1(a). A vortex-antivortex pair is induced in the nanoshell by a homogeneous dc magnetic field $\mathbf{B}_{\mathrm{dc}}$ parallel to the $z$-axis. An additional oscillating magnetic field $\mathbf{B}_{\mathrm{ac}}=\mathbf{e}_{x} B_{\omega} \cos (\omega t)$ with frequency $\omega$ and amplitude $B_{\omega} \ll B_{\mathrm{dc}}$ is applied along the $x$-axis. In our calculations we use spherical coordinates $r, \theta, \phi$, where $\theta=0$ corresponds to the positive direction of the $z$-axis in the Cartesian co-ordinate frame. We consider a spatially periodic $3 \mathrm{D}$ rectangular lattice of identical nanoshells, which are coupled to each other only electromagnetically. The unit cell of the lattice contains one nanoshell in the unit-cell center and has sizes $L_{x}, L_{y}, L_{z}>2 R$, so that the edges of the unit cell do not intersect with the nanoshell. The amplitude of the ac field is kept relatively small $\left(B_{\omega}<10^{-2}\right)$ so that the response of nanoshells to this field is linear.

To describe the vortex dynamics in nanoshells, we exploit the timedependent Ginzburg-Landau (TDGL) approach. Like in [21], the relevant quantities are made dimensionless by expressing lengths in units of $\sqrt{2} \xi$, time in units of $\pi \hbar /\left[4 k_{B}\left(T_{c}-T\right)\right] \approx 11.6 \tau_{G L}$, magnetic field in units of $\Phi_{0} /\left(4 \pi \xi^{2}\right)=H_{c 2} / 2$, current density in units of $\Phi_{0} /\left(2 \sqrt{2} \pi \mu_{0} \lambda^{2} \xi\right)$, and scalar potential in units of $2 k_{B}\left(T_{c}-T\right) /(\pi e)$. Here, $\Phi_{0}=\pi \hbar / e$ is the magnetic flux

quantum, $\mu_{0}$ is the vacuum permeability, $\lambda$ is the penetration depth, $\xi$ is the 

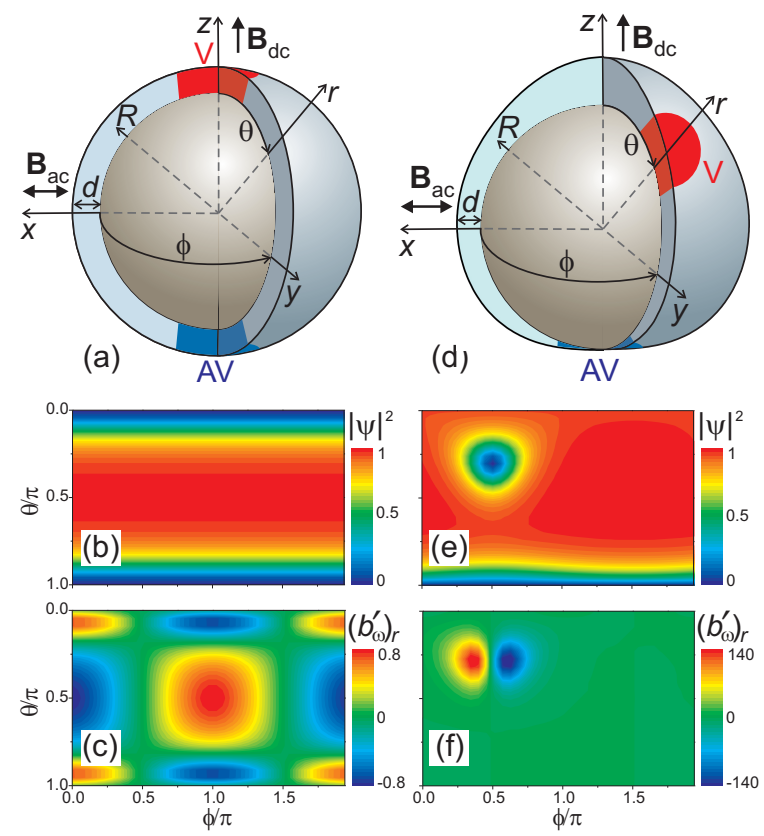

Figure 1: (Color online)(a) Sketch of a nanoshell with uniform thickness $d$. The red and blue shadow schematically show a vortex and an antivortex, respectively. (b) Distribution on the order parameter in a nanoshell with $R=2, d=0.35, \kappa=0.35$ for $L_{x}=L_{y}=L_{z}=$ $5.2, B_{\mathrm{dc}}=0.6$ and $B_{\mathrm{ac}}=0$. (c) Distribution of the radial component of the normalized magnetic field $\mathbf{b}_{\omega}^{\prime}$ in a nanoshell with $R=2, d=0.35, \kappa=0.35$ for $L_{x}=L_{y}=L_{z}=5.2$, $B_{\mathrm{dc}}=0.6, B_{\omega}=0.003, \omega=0.003$. Panels (d) to (f): same as in panels (a) to (c), respectively, but for the case of the nonuniform shell thickness described by Eq. (12) with $d_{0}=0.35, p=3$ and $q=5$. 
coherence length, $\tau_{G L}$ is the Ginzburg-Landau time, and $H_{c 2}$ is the second critical field.

We consider the case where the thickness of a superconducting shell is significantly smaller than the coherence length $\xi$, so that variations of the order-parameter magnitude across the shell as well as currents in the radial direction are negligible. In general, the thickness $d$ is assumed to be nonuniform. In this case, using the aforedescribed units the TDGL equation for the order parameter $\psi[22$ ], adapted to the case of spherical geometry, takes the form

$$
\begin{array}{r}
\left(\frac{\partial}{\partial t}+i \varphi\right) \psi=2 \psi\left(1-|\psi|^{2}\right) \\
+\frac{1}{d}\left(\frac{1}{R \sin \theta} \frac{\partial}{\partial \phi}-i A_{\phi}\right) d\left(\frac{1}{R \sin \theta} \frac{\partial}{\partial \phi}-i A_{\phi}\right) \psi \\
+\frac{1}{d}\left(\frac{1}{R} \frac{\partial}{\partial \theta}+\frac{\cot \theta}{R}-i A_{\theta}\right) d\left(\frac{1}{R} \frac{\partial}{\partial \theta}-i A_{\theta}\right) \psi,
\end{array}
$$

where $\varphi$ is the scalar potential and $\mathbf{A}$ is the vector potential.

The scalar-potential distribution is determined from the condition

$$
\nabla \cdot \mathbf{j}=0
$$

which reflects the continuity of currents in a superconducting shell. The total current density $\mathbf{j}$ in the shell is given by the sum of the normal and superconducting components:

$$
\begin{gathered}
\mathbf{j}=\mathbf{j}_{n}+\mathbf{j}_{s}, \\
\mathbf{j}_{n}=-\frac{\sigma}{2}\left(\nabla \varphi+\frac{\partial \mathbf{A}}{\partial t}\right), \\
\mathbf{j}_{s}=\operatorname{Im}\left(\psi^{*} \nabla \psi\right)-\mathbf{A}|\psi|^{2},
\end{gathered}
$$


where $\sigma$ is the normal-state conductivity, which is taken as $\sigma=1 / 12$ in our units (see Ref. [23]). The vector potential A, for which we choose the gauge $\nabla \cdot \mathbf{A}=0$, can be represented as

$$
\mathbf{A}=\mathbf{A}_{\mathrm{dc}}+\mathbf{A}_{\mathrm{ac}}+\mathbf{A}_{\mathrm{s}}
$$

Here $\mathbf{A}_{\mathrm{dc}}$ denotes the vector potential corresponding to the dc magnetic field $\mathbf{B}_{\mathrm{dc}}$, while $\mathbf{A}_{\mathrm{ac}}$ is related to the ac magnetic field. The vector potential $\mathbf{A}_{\mathrm{s}}$ describes the magnetic fields, induced by the currents $\mathbf{j}$, which flow in the superconducting shells:

$$
\mathbf{A}_{\mathbf{s}}(\mathbf{r})=\frac{1}{2 \pi \kappa^{2}} \int d^{3} r^{\prime} \frac{\mathbf{j}\left(\mathbf{r}^{\prime}\right)}{\left|\mathbf{r}-\mathbf{r}^{\prime}\right|}
$$

where $\kappa=\lambda / \xi$ is the Ginzburg-Landau parameter and integration is performed over the volume of the superconducting shells. The results, described below, are obtained for a fixed value of the Ginzburg-Landau parameter $(\kappa=0.35)$.

When solving numerically the TDGL equation (1), the gauge invariance of the discretized expressions is preserved by introducing link variables following the method of Refs. [24, 25]. Two-dimensional grids, used in our calculations, typically have $\gtrsim 30 R$ equally spaced nodes in the $\theta$-interval from 0 to $\pi$ and $\gtrsim 45 R$ equally spaced nodes in the $\phi$-interval from 0 to $2 \pi$, so that the distance between the neighboring nodes does not exceed $0.2 \xi$ (recall that the dimensionless parameter $R$ is given by the ratio of the nanoshell radius to $\sqrt{2} \xi)$. The step $h_{t}$ of the time variable $t$ is automatically adapted in the course of calculation. This adaptation is aimed to minimize the number of steps in $t$ and - at the same time - to keep the solving procedure accurate: the value of $h_{t}$ is chosen as large as possible provided that for $t_{\text {new }}=t_{\text {old }}+h_{t}$ 
the results, calculated with the steps $h_{t}$ and $h_{t} / 2$, are practically the same. Typically, the step $h_{t}$ is $\sim 10^{-5}$ to $\sim 10^{-3}$ depending on a specific distribution of the order parameter. For momentary distributions of the order parameter and the vector potential $\mathbf{A}$, an iteration procedure is used to determine from Eq. (2) the corresponding distribution of the scalar potential $\varphi$ with a relative accuracy not worse than $10^{-4}$. The (time-dependent) vector potential A and the corresponding link variables are calculated using Eq. (7), where the contributions of $\sim 10^{4}$ neighboring nanoshells are taken into account.

According to Ref. [26], the effective relative magnetic permeability, which corresponds to the response of a nanoshell lattice under consideration to the ac magnetic field $\mathbf{B}_{\mathrm{ac}}=\mathbf{e}_{x} B_{\omega} \cos \omega t$, can be expressed as

$$
\mu_{\omega}=\frac{B_{\omega}}{B_{\omega}+\left\langle B_{\mathrm{s} \omega}^{(c)}\right\rangle_{x}+i\left\langle B_{\mathrm{s} \omega}^{(s)}\right\rangle_{x}},
$$

where the numerator originates from the ac $B_{x}$-field, averaged over a unit-cell face, normal to the direction of the applied ac field, while the denominator is determined by the total ac $H_{x}$-field, averaged over a unit-cell edge parallel to the applied ac field, times $\mu_{0}$. Here the quantities

$$
\mathbf{B}_{\mathrm{s} \omega}^{(c)}=\frac{\omega}{\pi} \int_{t}^{t+2 \pi / \omega} d t^{\prime} \mathbf{B}_{\mathrm{s}}\left(t^{\prime}\right) \cos \left(\omega t^{\prime}\right)
$$

and

$$
\mathbf{B}_{\mathrm{s} \omega}^{(s)}=\frac{\omega}{\pi} \int_{t}^{t+2 \pi / \omega} d t^{\prime} \mathbf{B}_{\mathrm{s}}\left(t^{\prime}\right) \sin \left(\omega t^{\prime}\right)
$$

with $\mathbf{B}_{\mathrm{s}}=\nabla \times \mathbf{A}_{\mathrm{s}}$, describe the in-phase and out-of-phase magnetic field, respectively, induced by the superconducting nanoshells at frequency $\omega$. As 
seen from Eq. (7), these fields are inversely proportional to $\kappa^{2}$. By introducing the normalized complex field $\mathbf{b}_{\omega}$ with the real part $\mathbf{b}_{\omega}^{\prime}=\mathbf{B}_{\mathrm{s} \omega}^{(c)} / B_{\omega}$ and the imaginary part $\mathbf{b}_{\omega}^{\prime \prime}=\mathbf{B}_{\mathrm{s} \omega}^{(s)} / B_{\omega}$, equation (8) simplifies into

$$
\mu_{\omega}=\left(1+\left\langle b_{\omega}\right\rangle_{x}\right)^{-1}
$$

\section{Results and discussion}

In Fig. 1(b), we plot the equilibrium distribution of the order parameter in a nanoshell with radius $R=2$ and uniform thickness $d=0.35$ at $B_{\mathrm{dc}}=0.6$ and $B_{\mathrm{ac}}=0$. This distribution corresponds to a single vortexantivortex pair with the vortex (antivortex) pinned by the Magnus-Lorentz force to the northern (southern) pole of the nanoshell. In response to an ac magnetic field with $B_{\omega}=0.003, \omega=0.003$, applied to a cubic lattice of those nanoshells with $L_{x}=L_{y}=L_{z}=5.2$, the nanoshells induce their own magnetic field. The radial component of the corresponding normalized field $\mathbf{b}_{\omega}^{\prime}$ in a nanoshell is shown in Fig. 1(c). In the equatorial regions of the nanoshell, the magnitude and the sign of the field $\left(b_{\omega}^{\prime}\right)_{r}$ are mainly determined by the diamagnetic Meissner currents, induced in the nanoshell by the applied field $\mathbf{B}_{\mathrm{ac}}$. The distribution of $\left(b_{\omega}^{\prime}\right)_{r}$ near the poles is governed by the oscillatory motion of the vortex and antivortex. Indeed, the field $\mathbf{B}_{\mathrm{ac}}$, parallel to the $x$-axis, tends to shift the (anti)vortex from its equilibrium position on the $z$-axis towards the $x$-axis. Importantly, each (anti)vortex carries a relatively large magnetic moment, which is (anti)parallel to the $r$-axis. The local magnetic fields created by a vortex (antivortex) in the nanoshell and its vicinity are comparable in magnitude to the relatively strong dc field $B_{\mathrm{dc}}$. Therefore, oscillations of (anti)vortices can produce an appreciably high ac field. 


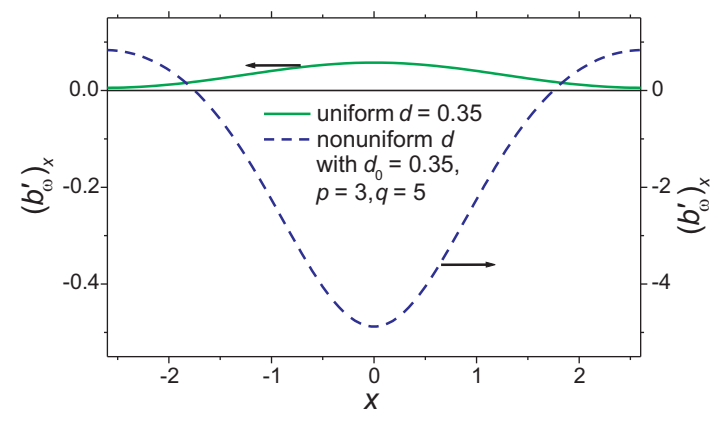

Figure 2: (Color online) Distribution of the $x$-component of the normalized magnetic field $\mathbf{b}_{\omega}^{\prime}$ along a unit-cell edge, parallel to the $x$-axis, for nanoshells of uniform thickness $d=0.35$ (solid line) and nonuniform thickness described by Eq. (12) with $d_{0}=0.35, p=3$ and $q=5$ (dashed line). The calculations correspond to $R=2, \kappa=0.35, L_{x}=L_{y}=L_{z}=5.2$, $B_{\mathrm{dc}}=0.6, B_{\omega}=0.003$, and $\omega=0.003$.

However, due to strong pinning of (anti)vortices to the nanoshell poles, the oscillation amplitude of (anti)vortices in the nanoshell lattice under consideration is rather small. As a result, the normalized magnetic field $\left(b_{\omega}^{\prime}\right)_{x}$ on the unit-cell edge parallel to the $x$-axis (see the solid curve in Fig. (2)) is actually dominated by the contribution of the Meissner currents. Consequently, the effective magnetic permeability of the nanoshell lattice $\mu_{\omega}^{\prime} \approx 0.915$, determined by Eq. (11), corresponds to a diamagnetic medium.

For nanoshells with a nonuniform thickness, the situation can become significantly different from that described above. Here we restrict ourselves to the case when the shell thickness $d$ varies according to the formula

$$
d=d_{0}\left\{\begin{array}{cc}
\left(1+\cos ^{p} \theta\right) & \text { for } \theta \leq \pi / 2 \\
\left(1-|\cos \theta|^{q}\right) & \text { for } \theta>\pi / 2
\end{array}\right.
$$

with $p, q>2$, as it is schematically shown in Fig. 1(d). Those variations of $d$ enhance the anivortex pinning at the southern pole of a nanoshell and, 
at the same time, tend to expel the vortex from the northern pole. If these variations are sufficiently large, the equilibrium position of the vortex in an isolated nanoshell corresponds to a ring-like potential well at $\theta \neq 0$. Since this potential well has perfect $C_{\infty}$ symmetry, the vortex can easily move within the well (in the $\phi$ direction) when applying even a very weak additional force, caused by the field $\mathbf{B}_{\mathrm{ac}}$. In a rectangular nanoshell lattice the $C_{\infty}$ symmetry of the potential well for a vortex is somewhat violated, mainly due to the magnetic field of the Meissner currents induced by the field $\mathbf{B}_{\mathrm{dc}}$ in the neighboring nanoshells. In such a lattice, subjected to a weak ac magnetic field parallel to the $x$-axis, the vortex typically oscillates around $\phi=\pi / 2$, as illustrated by Figs. 1(e) and (f), or around $\phi=3 \pi / 2$. At relatively low frequencies $\omega$ the amplitude of the vortex oscillations can be rather large, and the radial ac magnetic field induced by these oscillations appears by orders of magnitude stronger than the applied field $B_{\omega}$ [see Fig. $\left.1(f)\right]$. The ac field, induced by the nanoshells on the unit-cell edge, parallel to the $x$-axis, is fully dominated by the contribution of the oscillating vortices. In the central part of the unit-cell edge the $x$-component of this field is opposite to the applied ac field and significantly exceeds it $\left(\left|\left(b_{\omega}^{\prime}\right)_{x}\right|>1\right.$; see the dashed curve in Fig. 2). so that the total normalized field $1+\left(b_{\omega}^{\prime}\right)_{x}$ becomes negative.

In Fig. 3 (a) the $x$-components of the normalized magnetic fields $\mathbf{b}_{\omega}^{\prime}$ and $\mathbf{b}_{\omega}^{\prime \prime}$ averaged over the unit-cell edge, parallel to the $x$-axis, are plotted as a function of $\omega$. As seen from Fig. 3(a), at $\omega<0.04$ the averaged field $\left\langle b_{\omega}^{\prime}\right\rangle_{x}$ is negative. Moreover, for $\omega<0.004$ its magnitude is larger than 1 , so that the real part of the effective magnetic permeability of the nanoshell lattice $\mu_{\omega}^{\prime}$, defined by Eq. (11), appears negative [see Fig. 3(b)]. As further seen from 
Fig. 3, in the low-frequency range the out-of-phase field component $\left\langle b_{\omega}^{\prime \prime}\right\rangle_{x}$ and, consequently, the imaginary part of $\mu_{\omega}$ rapidly decrease when decreasing $\omega$. This decrease originates from a reduction of the energy dissipation by the vortices, which move with smaller average velocities at lower frequencies. At relatively high frequencies, the amplitude of the vortex oscillations and their contribution to $\left\langle b_{\omega}\right\rangle_{x}$ and $\mu_{\omega}$ diminish with increasing $\omega$. For $\omega>0.01$ the magnetic response of the nanoshell lattice is dominated by the contribution of the dissipationless Meissner currents, induced by the field $\mathbf{B}_{\mathrm{ac}}$. In this frequency range the effective permeability $\mu_{\omega}$ corresponds to a diamagnetic medium, and the imaginary part of $\mu_{\omega}$ gradually vanishes with increasing $\omega$.

Interestingly, the effective ac magnetic permeability of a nanoshell array is sensitive not only to the relevant geometric and material parameters and the ac-field frequency but also to the magnitude of the applied dc field $B_{\mathrm{dc}}$. In this connection, it seems worth mentioning that in nanoshells with $R \sim 2$ the state with one vortex-antivortex pair appears (meta)stable in a rather wide range of $B_{\mathrm{dc}}$ [14]. As illustrated in Fig. 4, the ac magnetic response of a nanoshell array at a fixed, relatively low frequency can be efficiently controlled by simply varying the dc field $B_{\mathrm{dc}}$ within this range. Indeed, the plots in Fig. 4(a) show that with increasing $B_{\mathrm{dc}}$ the magnitude of the negative normalized field $\left\langle b_{\omega}^{\prime}\right\rangle_{x}$ changes from relatively large values $\left|\left\langle b_{\omega}^{\prime}\right\rangle_{x}\right|>1$ to values $\left|\left\langle b_{\omega}^{\prime}\right\rangle_{x}\right|<1$. This behavior can be explained by a shift of the equilibrium vortex position towards the nanoshell pole and the corresponding decrease of the angle between the vortex magnetic moment and the $z$-axis when applying a higher dc field $B_{\mathrm{dc}}$. As seen from Fig. 4(a), this leads to 


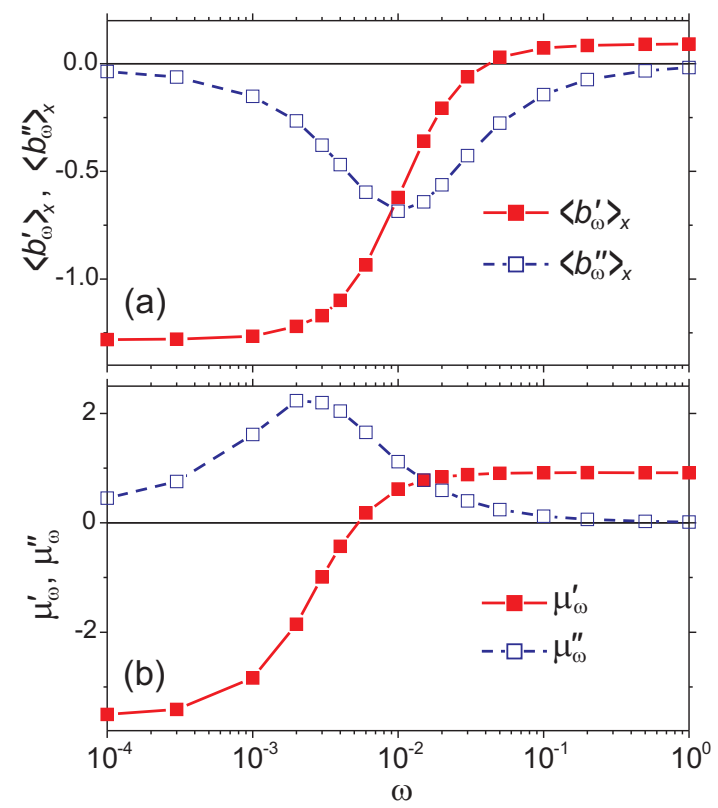

Figure 3: (Color online) (a) $x$-components of the normalized magnetic fields $\mathbf{b}_{\omega}^{\prime}$ and $\mathbf{b}_{\omega}^{\prime \prime}$ averaged over the unit-cell edge, parallel to the $x$-axis, as a function of the applied ac field frequency.(b) Real and imaginary parts of the effective magnetic permeability of the array of nanoshells as a function of the applied ac field frequency. The calculations correspond to $R=2, d_{0}=0.35, p=3, q=5, \kappa=0.35, L_{x}=L_{y}=L_{z}=5.2, B_{\mathrm{dc}}=0.8$, and $B_{\omega}=0.001$. 


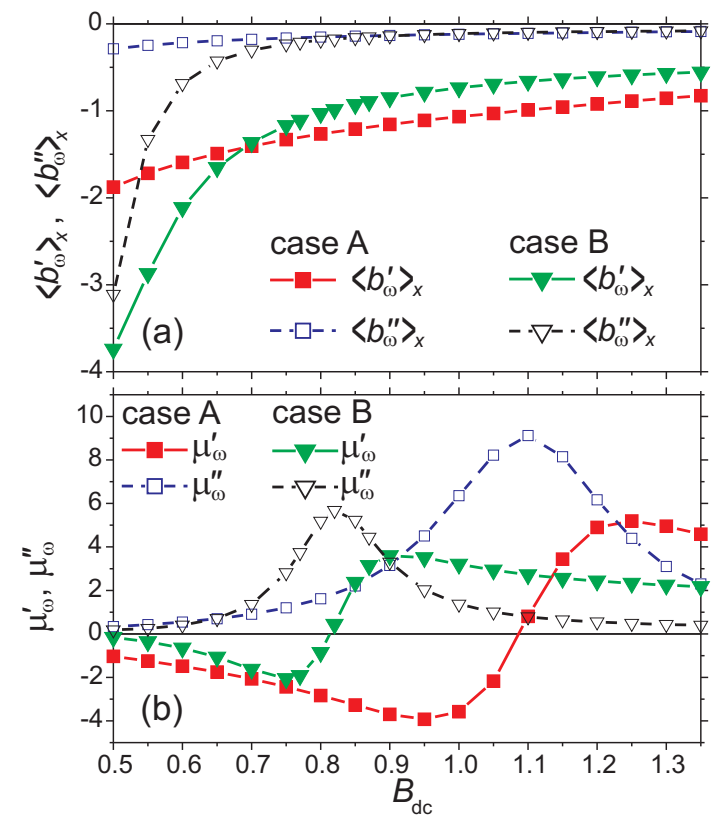

Figure 4: (Color online) Averaged $x$-components of the normalized magnetic fields $\mathbf{b}_{\omega}^{\prime}$ and $\mathbf{b}_{\omega}^{\prime \prime}$ (a) and real and imaginary parts of the corresponding effective magnetic permeability $\mu_{\omega}$ (b) as a function of the applied dc magnetic field. The calculations are performed for $L_{x}=L_{y}=L_{z}=5.2, p=3, q=5, \omega=0.001$ (case A) and $L_{x}=L_{y}=4.4, L_{z}=6$, $p=q=5, \omega=0.003$ (case B). Other relevant parameters are $R=2, d_{0}=0.35, \kappa=0.35$, $B_{\omega}=0.001$.

an evolution of the ac magnetic permeability $\mu_{\omega}^{\prime}$ from negative values at lower dc fields $B_{\mathrm{dc}}$ to relatively large positive values, which correspond to (super)paramagnetic ac response of the nanoshell lattice at higher fields $B_{\mathrm{dc}}$.

\section{Conclusions}

Using the time-dependent Ginzburg-Landau approach, we have investigated vortex states in superconducting spherical nanoshells with nonuniform 
thickness, subjected to a homogeneous dc magnetic field, and analyzed the vortex dynamics in the presence of an additional weak ac magnetic field, perpendicular to the dc field. It is shown that by increasing the shell thickness at the pole, determined by the direction of the dc field, the equilibrium vortex position can be shifted from this pole to the surrounding ring-like potential well. In this state, the amplitude of vortex oscillations in an ac magnetic field appears strongly enhanced, so that the magnetic response of a nanoshell is fully dominated by the contribution of the oscillating magnetic moment of the vortex. As distinct from the case of nanoshells with uniform thickness, which are typically characterized by a diamagnetic ac response, for a 3D lattice made of nanoshells with nonuniform thickness the real part of the effective ac magnetic permeability can take negative values at sufficiently low frequencies. We have demonstrated that the ac magnetic response of such a lattice can be efficiently controlled by the magnitude of the applied dc magnetic field. By varying this dc field the real part of the effective ac magnetic permeability of the nanoshell lattice can be tuned in a wide range: from negative values to relatively large positive ("superparamagnetic") values.

\section{Acknowledgements}

This work was supported by Methusalem funding by the Flemish government, the Flemish Science Foundation (FWO-Vl), in particular FWO projects G.0365.08, G.0370.09N, , G.0119.12N, and G.0115.12N, the Scientific Research Community project WO.033.09N, the Belgian Science Policy, and the ESF NES network. 


\section{References}

[1] Y. Liu, Yu. Zadorozhny, M. M. Rosario, B. Y. Rock, P. T. Carrigan, and H. Wang, Science 294 (2001) 2332.

[2] H. Wang, M. M. Rosario, N. A. Kurz, B. Y. Rock, M. Tian, P. T. Carrigan, and Y. Liu, Phys. Rev. Lett. 95 (2005) 197003.

[3] M. Lu-Dac and V. V. Kabanov, Phys. Rev. Lett. 105 (2010) 157005.

[4] Y. Chen, A. A. Shanenko, and F. M. Peeters, Phys. Rev. B 81 (2010) 134523.

[5] P. Sabatino, G. Carapella and G. Costabile, Supercond. Sci. Technol. 24 (2011) 125007.

[6] V. M. Fomin, R. O. Rezaev, and O. G. Schmidt, Nano Lett. 12 (2012) 1282.

[7] A. Müller, S. E. C. Dale, M. A. Engbarth, S. J. Bending, L. M. Peter, A. Knittel, and H. Fangohr, Adv. Funct. Mater. 21 (2011) 1874.

[8] A. R. de C. Romaguera, M. M. Doria, and F. M. Peeters, Phys. Rev. B 76 (2007) 020505(R).

[9] O. N. Shevtsova, Supercond. Sci. Tech. 21 (2008) 065010.

[10] B. Xu, M. V. Milošević, and F. M. Peeters, Phys. Rev. B 77 (2008) 144509.

[11] B. Xu, M. V. Milošević and F. M. Peeters, New J. Phys. 11 (2009) 013020. 
[12] Q. Du and L. Ju, J. Comput. Phys. 201 (2004) 511.

[13] Q. Du and L. Ju, Math. Comput. 74 (2004) 1257.

[14] V. N. Gladilin, J. Tempere, I. F. Silvera, J. T. Devreese, and V. V. Moshchalkov, Phys. Rev. B 77 (2008) 024512.

[15] J. Tempere, V. N. Gladilin, I. F. Silvera, J. T. Devreese, and V. V. Moshchalkov, Phys. Rev. B 79 (2009) 134516.

[16] M. M. Doria, A. R. de C. Romaguera, M. V. Milošević and F. M. Peeters, Europhys. Lett. 79 (2007) 47006.

[17] L. R. E. Cabral, J. Albino Aguiar, Physica C 470 (2010) 796.

[18] R. D. Averitt, D. Sarkar, and N. J. Halas, Phys. Rev. Lett. 78 (1997) 4217.

[19] S. J. Oldenburg, R. D. Averitt, S. L. Westcott, and N. J. Halas, Chem. Phys. Lett. 288 (1998) 243.

[20] Like in [14, 15], we use the term "vortex" ("antivortex") if the magnetic moment, induced by the corresponding circulating quasi-2D supercurrents, is parallel (antiparallel) to the outer normal to the superconductor shell.

[21] A. V. Silhanek, V. N. Gladilin, J. Van de Vondel, B. Raes, G. W. Ataklti, W. Gillijns, J. Tempere, J. T. Devreese and V. V. Moshchalkov, Supercond. Sci. Tech. 24 (2011) 024007. 
[22] S. J. Chapman, Q. Du, M. D. Gunzburger, Z. Angew. Math. Phys. 47, 410 (1996).

[23] R. Kato, Y. Enomoto, S. Maekawa, Phys. Rev. B 44 (1991) 6916.

[24] R. Kato, Y. Enomoto, S. Maekawa, Phys. Rev. B 47 (1993) 8016.

[25] W. D. Gropp, H. G. Kaper, G. K. Leaf, D. M. Levine, M. Palumbo, and V. M. Vinokur, J. Comp. Phys 123 (1996) 254.

[26] J. B. Pendry, A. J. Holden, D. J. Robbins, and W. J. Stewart, IEEE Trans. Microwave Theory Tech. 47 (1999) 2075. 